\title{
BMJ Open Leg ischaemia management collaboration (LIMb): study protocol for a prospective cohort study at a single UK centre
}

\author{
John S M Houghton, ${ }^{\oplus 1,2,3}$ Sarah Nduwayo, , ${ }^{1,2,3}$ Andrew T O Nickinson, ${ }^{01,2,3}$ \\ Tanya J Payne, ${ }^{1,3}$ Sue Sterland, ${ }^{1,3}$ Mintu Nath, ${ }^{1,3}$ Laura J Gray, ${ }^{\bullet} 4$ \\ Greg S McMahon, ${ }^{1,2}$ Harjeet S Rayt, ${ }^{1,2}$ Sally J Singh, ${ }^{5}$ Thompson G Robinson, ${ }^{\odot 1,3}$ \\ Simon P Conroy, ${ }^{\circ}$ Victoria J Haunton, ${ }^{1}$ Gerry P McCann, ${ }^{\circledR} 1,3$ \\ Matthew J Bown, ${ }^{1,2,3}$ Robert S M Davies, ${ }^{\oplus 1,2}$ Rob D Sayers ${ }^{1,2,3}$
}

To cite: Houghton JSM, Nduwayo S, Nickinson ATO, et al. Leg ischaemia management collaboration (LIMb): study protocol for a prospective cohort study at a single UK centre. BMJ Open 2019;9:e031257. doi:10.1136/ bmjopen-2019-031257

- Prepublication history and additional material for this paper are available online. To view these files, please visit the journal online (http://dx.doi. org/10.1136/bmjopen-2019031257).

Received 25 April 2019

Revised 26 July 2019

Accepted 29 July 2019

Check for updates

(c) Author(s) (or their employer(s)) 2019. Re-use permitted under CC BY-NC. No commercial re-use. See rights and permissions. Published by BMJ.

For numbered affiliations see end of article.

Correspondence to Mr John S M Houghton; john.houghton@nhs.net

\section{ABSTRACT}

Introduction Severe limb ischaemia (SLI) is the end stage of peripheral arterial occlusive disease where the viability of the limb is threatened. Around $25 \%$ of patients with SLI will ultimately require a major lower limb amputation, which has a substantial adverse impact on quality of life. A newly established rapid-access vascular limb salvage clinic and modern revascularisation techniques may reduce amputation rate. The aim of this study was to investigate the 12-month amputation rate in a contemporary cohort of patients and compare this to a historical cohort. Secondary aims are to investigate the use of frailty and cognitive assessments, and cardiac MRI in risk-stratifying patients with SLI undergoing intervention and establish a biobank for future biomarker analyses.

Methods and analysis This single-centre prospective cohort study will recruit patients aged 18-110 years presenting with SLI. Those undergoing intervention will be eligible to undergo additional venepuncture (for biomarker analysis) and/or cardiac MRI. Those aged $\geq 65$ years and undergoing intervention will also be eligible to undergo additional frailty and cognitive assessments. Follow-up will be at 12 and 24 months and subsequently via data linkage with NHS Digital to 10 years postrecruitment. Those undergoing cardiac MRI and/or frailty assessments will receive additional follow-up during the first 12 months to investigate for perioperative myocardial infarction and frailty-related outcomes, respectively. A sample size of 420 patients will be required to detect a $10 \%$ reduction in amputation rate in comparison to a similar sized historical cohort, with $90 \%$ power and $5 \%$ type I error rate. Statistical analysis of this comparison will be by adjusted and unadjusted logistic regression analyses.

Ethics and dissemination Ethical approval for this study has been granted by the UK National Research Ethics Service (19/L0/0132). Results will be disseminated to participants via scientific meetings, peer-reviewed medical journals and social media.

Trial registration number NCT04027244.

\section{Strengths and limitations of this study}

- This prospective study of a contemporary cohort of patients with severe limb ischaemia (including both patients with and without diabetes) will provide a valuable update to observational evidence.

- The inclusion of patients lacking capacity reduces potential selection bias.

- The use of comprehensive assessments of frailty syndromes and cardiac MRI may provide valuable additional strategies to assess risk.

- The single-centre study design will limit the generalisability of its results.

- The retrospective collection of historical control data and observational nature of the study will limit the strength of evidence of the results.

\section{INTRODUCTION}

Peripheral arterial occlusive disease (PAOD) is a chronic condition caused by atherosclerosis of arteries supplying the lower limbs. In high-income countries, its prevalence is over $10 \%$ in those aged over 65 years. ${ }^{1}$ PAOD is often asymptomatic, but as the disease progresses, patients may develop symptoms such as intermittent claudication. ${ }^{2}$ Severe limb ischaemia (SLI) is the end stage of PAOD in which the viability of the limb is threatened due to the degree of arterial disease and subsequent ischaemia in the peripheral tissues, and is characterised by ischaemic rest pain and tissue necrosis, leading to ulceration and gangrene. ${ }^{3}$ Treatment options include open surgical and endovascular revascularisation, with or without amputation of toes, surgical drainage of infection and debridement of infected/necrotic tissue. In some patients, revascularisation is not possible and 
primary major lower limb amputation, or amputation after failed attempted revascularisation, is required. ${ }^{4}$

Lower limb amputation is a life-changing tragedy for the patient and a huge financial burden to the National Health Service (NHS). Between 2003 and 2013, over 4000 major lower limb amputations were performed each year in England alone. ${ }^{5}$ With the rising prevalence of diabetes and diabetic foot disease (DFD), a diabetes-related major lower limb amputation is performed every $30 \mathrm{~s}$ worldwide and over 7000 diabetes-related major and minor amputations are now performed each year in England and Wales. ${ }^{67}$ Major lower limb amputation negatively affects the quality of life due to its significant impact on mobility, independence, social isolation and ability to carry out activities of daily living. ${ }^{8}$ Only around 20\% of those undergoing an amputation for PAOD are independently ambulant 1 year postprocedure. ${ }^{9} 10$

\section{Frailty, cognitive impairment and delirium}

A major challenge in managing patients with SLI is decision-making regarding optimum treatment strategies. As well as anatomical considerations regarding revascularisation strategy, an accurate assessment of both the risk of treatment failure and the morbidity associated with a procedure should be taken into account. This is particularly challenging in patients with SLI as they are often elderly with multiple comorbidities. ${ }^{11}$ Frailty is a distinct health state and is increasingly recognised as a major contributor to adverse outcomes in surgical patients. ${ }^{12}$ Frailty is defined as 'a medical syndrome with multiple causes and contributors that is characterised by diminished strength, endurance, and reduced physiologic function that increases an individual's vulnerability for developing increased dependency and/or death'. ${ }^{13}$ Around $10 \%$ of people aged over 65 years are frail, increasing to more than $30 \%$ of those aged over 85 years, while prevalence of frailty in patients with SLI may be over 50\% ${ }^{111415}$ Frail patients are less able to respond to acute stressors, such as major surgery, and are therefore more susceptible to adverse health outcomes such as mortality and postoperative complications. ${ }^{1617}$ Multiple validated tools used to assess patients for frailty have been developed which can be used in preoperative assessment. ${ }^{1318}$

Both cognitive impairment and sarcopenia are also of interest in this cohort as they are associated with frailty, vascular disease and vascular risk factors. ${ }^{19-21}$ Cognitive impairment, encompassing a spectrum of diseases from mild cognitive impairment to dementia, manifests as memory problems, poor concentration and difficulty making decisions about things affecting everyday life. Sarcopenia is a "progressive and generalised skeletal muscle disorder that is associated with increased likelihood of adverse outcomes' and is defined as low muscle strength plus low muscle quantity or quality and/or low physical performance. ${ }^{22}$ Sarcopenia has been assessed both clinically and radiologically (using cross-sectional imaging) in vascular patients. ${ }^{23}$ Frailty, cognitive impairment and sarcopenia have been implicated in worse outcome following vascular surgery. ${ }^{17}$ Postoperative delirium is one such outcome which has been shown to be associated both with frailty in vascular patients and worse short-term and long-term outcome following vascular surgery. ${ }^{25} 26$

\section{Coronary artery disease}

Coronary artery disease (CAD) is a highly prevalent and major comorbidity of patients with PAOD. Very few vascular surgery patients have normal coronary arteries on coronary angiography. ${ }^{27}$ Coronary angiography has also shown that around $60 \%$ of vascular surgery patients have severe $\mathrm{CAD}$ (>70\% stenosis), and in those without symptoms or history of ischaemic heart disease undergoing vascular surgery, around $70 \%$ have evidence of CAD, half of whom have severe disease ${ }^{27}{ }^{28}$ Patients with PAOD have a $20 \%-60 \%$ increased risk for myocardial infarction (MI) and a twofold to sixfold increased risk of death due to CAD-related events. ${ }^{29}$ Perioperative MI (based on elevated troponin) has been reported in up to $24 \%$ of vascular patients, with $82 \%$ of these not exhibiting symptoms. ${ }^{30}$

Cardiac MRI is the most sensitive imaging modality for the detection of myocardial necrosis/infarct and is highly accurate for the detection of reversible ischaemia in a single examination. ${ }^{31-34}$ Modern software allows images to be acquired completely free-breathing, using motion-correction techniques and highly accelerated sequences, which is particularly important for the frail or breathless patient. ${ }^{356}$ In addition, myocardial blood flow can be quantified fully automatically within minutes of acquisition, allowing measurement of stress and rest myocardial blood flow and perfusion reserve. ${ }^{37}$ The identification of significant cardiac disease in these patients may allow further risk assessment and stratification to ensure that each patient has an optimum treatment plan.

\section{RATIONALE}

The most widely cited estimates of outcomes in patients with SLI are from the Trans-Atlantic Consensus Statement II (2007) which states that $50 \%$ will initially undergo revascularisation and $25 \%$ primary amputation, and at 1 year, $30 \%$ will be alive and have had an amputation, while $25 \%$ will have died. ${ }^{4}$ In recent years, there has been a marked demographic shift with increasing prevalence of diabetes among patients with SLI and intervention in older, frailer patients. ${ }^{1138}$ While there has been little change in recommended medical therapy of PAOD, ${ }^{4} 39$ new endovascular technologies and improved techniques such as ultradistal bypass and covered endovascular revascularisation of aortic bifurcation allow increasingly aggressive revascularisation strategies. ${ }^{40-42}$ The Leicester Vascular Institute (based at Glenfield Hospital, University Hospitals of Leicester NHS Trust) is a tertiary referral vascular unit providing all vascular services for Leicestershire and Rutland, compromising a diverse population of approximately 1.1 million. Both a recently established 
rapid-access limb salvage clinic and direct vascular admission for all patients admitted as an emergency for DFD aim to improve outcomes in patients with SLI and ultimately reduce the need for major lower limb amputation in line with recent guidelines from The Vascular Society of Great Britain and Ireland. ${ }^{43}$ A contemporary cohort study will provide useful evidence of outcomes in the context of current SLI demographics, revascularisation strategies and improved care pathways. Additionally, this prospective cohort study provides an opportunity to make a detailed assessment of frailty, cognitive impairment and ischaemic heart disease (as detected by cardiac MR) in patients with SLI, as well as establish a biobank for future biomarker analysis, to establish each of their roles in risk-stratifying patients undergoing intervention for SLI.

\section{STUDY AIMS}

\section{Primary aim}

The primary aim of this study is to determine the proportion of patients with SLI undergoing major lower limb amputation within 12 months of presentation. The primary hypothesis is that contemporary amputation rate is lower than historical data.

\section{Secondary aims}

Secondary aims are to assess clinically important shortterm ( $\leq 3$ months), medium-term (3-12 months) and long-term ( $>12$ months) outcomes in those undergoing and not undergoing amputation. We also aim to determine prevalence and degree of frailty, cognitive impairment and cardiac disease (detected by stress MRI), as well as establish a biobank for future analysis of biomarkers, in those patients undergoing procedures for SLI. This may allow risk stratification and also to investigate whether patients with a higher risk of poor perioperative outcome or long-term worse prognosis are undergoing procedures with increased risk of morbidity and mortality.

\section{METHODS AND ANALYSIS \\ Study design}

This study will be a single-centre prospective observational cohort study undertaken at the Leicester Vascular Institute. Recruitment will continue for a period of 2 years with a total of 10-year follow-up.

\section{Primary outcome measure}

The primary outcome measure of the study is major amputation rate.

\section{Secondary outcome measures}

The secondary outcome measures for short-term, medium-term and long-term follow-up data include, but not limited to, quality of life, functional performance, anxiety and depression, morbidity, mortality and amputation-free survival.

\section{STUDY PARTICIPANTS AND ELIGIBILITY}

\section{Eligibility and consent}

All patients with SLI attending the Leicester Vascular Institute will be screened for their eligibility. Those eligible will be given verbal and written information detailing the nature of the study and time to decide whether they want to participate. Patients will be eligible for inclusion up until the point they undergo an intervention. Full written informed consent will be taken for patients agreeing to participate in the study. Consent will be gained to allow investigators to undertake additional assessments not routinely performed in this cohort of patients, as well as to review medical records for both baseline data and outcomes measured at various points during the 24 months following recruitment. Consent will also be taken for data linkage with NHS Digital records for long-term outcomes at 10 years postrecruitment.

The researchers will have primary responsibility for assessing the capacity of the individual to participate in research according to the Mental Capacity Act 2005. Given the low risk and burden of this study to patients, it is reasonable to seek consultee consent in patients who do not have capacity. A 'hierarchy' of consent will be followed; this approach to the enrolment process has successfully been used in recent NIHR-funded studies. ${ }^{44}$

1. Potential patients with capacity are recruited, provided they are willing to provide consent.

2. Where patients do not have capacity, they may be recruited using consultee agreement (such as an unpaid carer or next of kin) under Section 31(2) of the Mental Capacity Act 2005.

3. Checks will be made with the potential patient's general practitioner or hospital records that there is no relevant advance decision relating to research under Section 33(2).

4. We will use a consultee under Section 32(3) whenever possible.

Personal consultees will be provided with verbal and written information detailing their role and the nature of the study. They will be given time to decide whether they feel the patient would want to participate in the study and have an opportunity to ask questions prior to completing the personal consultee declaration form. Both the clinical and the research team will endeavour to identify a potential personal consultee for any eligible patient that lacks capacity; however, if one cannot be identified, the patient will not be invited to participate in the study.

\section{Primary cohort}

All patients attending the Leicester Vascular Institute with a diagnosis of SLI will be considered for inclusion to the study. SLI is defined as pain at night or at rest and/ or ulceration or gangrene in the affected $\operatorname{limb}(\mathrm{s})$ for a minimum of 2 weeks attributed to confirmed PAOD. ${ }^{45}$

\section{Historical controls}

A retrospective cohort of historical controls with SLI will be identified for a 24-month period (2013-2015) prior to 
establishing the Leicester Vascular Institute at the Glenfield Hospital site. Approximately 420 patients with a primary admission diagnosis of SLI will be identified from local coding data using the International Classification of Diseases-10 code I70.2. Hospital paper and electronic records will be interrogated to identify baseline characteristics and outcomes including major amputation. This will be cross-referenced against Trust Hospital Episode Statistics data for major amputations (OPCS-4 code X09) to ensure accuracy.

\section{Additional assessments}

Patients recruited to the primary cohort who are offered intervention (minor amputation/debridement, revascularisation or primary major amputation) will also be given the opportunity to participate in additional assessments, depending on them meeting the inclusion/exclusion criteria. These additional assessments will investigate frailty and cognitive impairment, and cardiac dysfunction on short-term and medium-term outcomes. Patients undergoing intervention will also be eligible for venepuncture to collect serum and plasma samples to establish a biobank for future biomarker analyses. Patients who are treated medically with no planned intervention will be eligible for recruitment to the primary cohort only.

Full inclusion and exclusion criteria are detailed in table 1 .

\section{STUDY PROCEDURES}

A study overview with potential recruitment numbers (based on local audit data 2018/2019) is detailed in figure 1. Eligible patients may be recruited to multiple additional assessments dependent on their consent.

\section{Primary cohort}

Baseline data will be collected from the standard admission proforma as part of routine attendance at the Leicester Vascular Institute, which includes:

- Presenting complaint.

Table 1 Inclusion and exclusion criteria

\section{Primary cohort}

Inclusion criteria:

- Patients presenting to the Leicester Vascular Institute with SLI

- Patients aged 18-110 years

\section{Exclusion criteria:}

- SLI not caused by PAOD

- Patients undergoing intervention during their index presentation prior to recruitment

- Patients lacking capacity with no accompanying next of kin, relative, partner or friend who can act as a personal consultee

- Patients who cannot read, write or understand English

- Any significant disease or disorder which may either put the patients at risk because of participation in the study or may influence the results of the study or the patient's ability to participate in the study

\section{Frailty and cognitive impairment additional assessments \\ Inclusion criteria: \\ Exclusion criteria: \\ Patients recruited to the primary cohort in whom a decision $>$ No additional exclusion criteria has been made to undergo an intervention for SLI \\ Patients aged $>65$ years}

\section{Cardiac MRI additional assessments}

Inclusion criteria:

Exclusion criteria:

- Patients recruited to the primary cohort in whom a decision has been made to undergo an intervention for SLI

- Absolute contraindications to cardiac MRI:

- Pregnancy; non-MR safe PPM/ICD; non-MR safe intraauricular implants; non-MR safe intracranial clips; severe claustrophobia; unstable angina

- Contraindication to gadolinium contrast agent:

- Known adverse reaction; chronic renal failure (eGFR <30 $\mathrm{mL} / \mathrm{min} / 1.73 \mathrm{~m}^{2}$ )

- Patients lacking capacity to consent for cardiac MRI

$\begin{array}{ll}\text { Biomarkers additional assessments } & \text { Exclusion criteria: } \\ \text { Inclusion criteria: } & \text { Patients recruited to the primary cohort in whom a decision } \\ \text { has been made to undergo an intervention for SLI } & \end{array}$

eGFR, estimated glomerular filtration rate; ICD, implantable cardioverter-defibrillator;MR(I), magnetic resonance (imaging); PAOD, peripheral arterial occlusive disease; PPM, permanent pacemaker; SLI, severe limb ischaemia. 


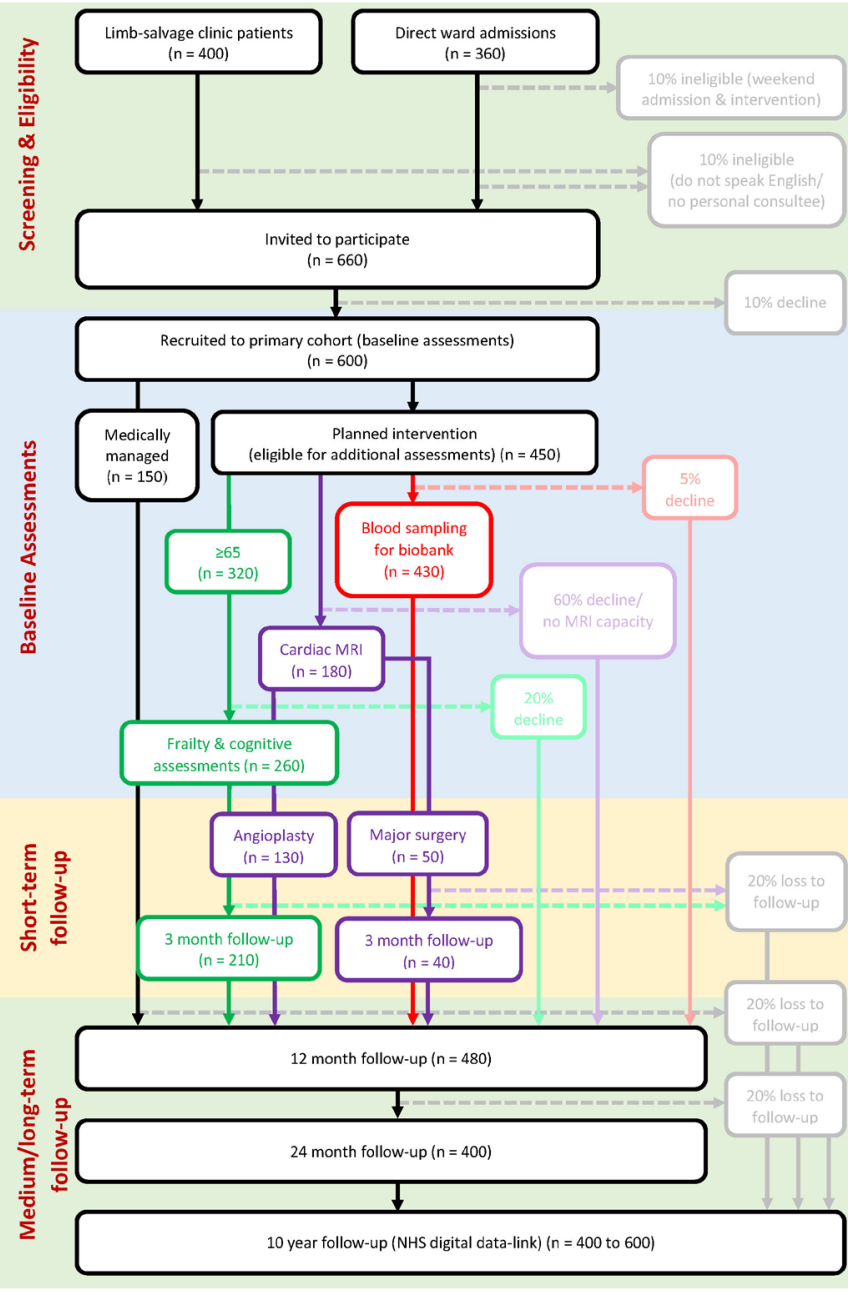

Figure 1 Study overview. Numbers are estimates for the whole 2-year recruitment period and based on local audit data from 2018/2019.

- Medical history/comorbidities.

- Social history (including smoking status).

- Current regular medications.

- Observations.

- Examination findings (lower limb pulses; Society of Vascular Surgery Wound, Ischaemia, foot Infection (WIfI) score ${ }^{38}$; Site, Ischaemia, Neuropathy, Bacterial infection, And Depth (SINBAD) score. ${ }^{46}$

- Ankle Brachial Pressure Index (ABPI).

- Lower limb arterial duplex report.

- Clinical Frailty Scale (CFS) ${ }^{47}$

- Vascular Quality of Life (VascuQol) Questionnaire. ${ }^{48}$ The following study data that will be collected in addition to routine clinic practice include:

- Barthel Activities of Daily Living (ADL) index. ${ }^{49}$

- Hospital Anxiety and Depression Scale (HADS). ${ }^{50}$

At 12 months ( \pm 1 month), patients will receive telephone follow-up to collect CFS, Barthel ADL index, HADS, VascuQol and limb salvage data. These same data will be collected again at 24 months $( \pm 1$ month). Electronic hospital records will be interrogated to collect data regarding procedures patients have undergone for SLI during the 12 months postrecruitment.

A long-term data-linkage process will be employed once the first patient has been recruited. This will provide mortality data for patients in the primary cohort and aid in project management (ie, not sending follow-up requests to deceased patients). After the 2-year follow-up period, patients in the primary cohort will be linked with NHS Digital records to provide long-term follow-up data for a total 10-year period following recruitment.

Frailty and cognitive impairment additional assessments

Additional data collected at baseline will include:

- Edmonton Frail Scale (EFS).$^{51}$

- Montreal Cognitive Assessment (MoCA). ${ }^{52}$

- Short Physical Performance Battery (SPPB) ${ }^{53}$

- Grip strength (bilateral) measured using a dynamometer.

- Single Question in Delirium (SQiD). ${ }^{54}$

- 4 'A's Test (4AT) for rapid delirium screening. ${ }^{55}$

At 24 and 72 hours postoperatively, patients will undergo screening for delirium using both the SQiD and 4AT. Patients who have been discharged from hospital prior to 24 and/or 72 hours postoperatively will undergo screening for delirium via telephone using the SQiD only (asking a relative or carer).

At 3 months ( \pm 1 month) and 12 months ( \pm 1 month), patients will attend a follow-up clinic where the following assessments will be undertaken: CFS, EFS, MoCA, SPPB, grip strength, Barthel ADL index, VascuQoL and HADS. No additional telephone follow-up will be required at 12 months as all relevant assessments will be undertaken during the 12-month clinic visit.

For patients who agree to participate in the frailty and cognitive impairment additional assessment, advanced consent will be gained to undertake delirium assessments postoperatively. It is possible that during the course of the follow-up period, patients may lose capacity before completing the study follow-up. Accordingly, advanced consent will be gained at the point of recruitment to undertake all the required assessments during follow-up. In the event that a patient loses capacity during the course of the study, a personal consultee will be sought to both confirm ongoing participation in the study and help complete the follow-up assessments.

\section{Cardiac MRI additional assessments}

The cardiac MR scan will be performed on a 3T scanner (Siemans Skyra or Vida) using a 32ch cardiac coil. The MRI protocol is outlined in figure 2 and will include ${ }^{56}$ :

i. Left ventricular (LV) function cine imaging

ii. Adenosine and/or dobutamine stress and rest perfusion imaging.

iii. Late gadolinium enhancement.

iv. If time permits and the patient is able to tolerate the scan, additional sequences such as aortic distensibility and angiography may be performed. 


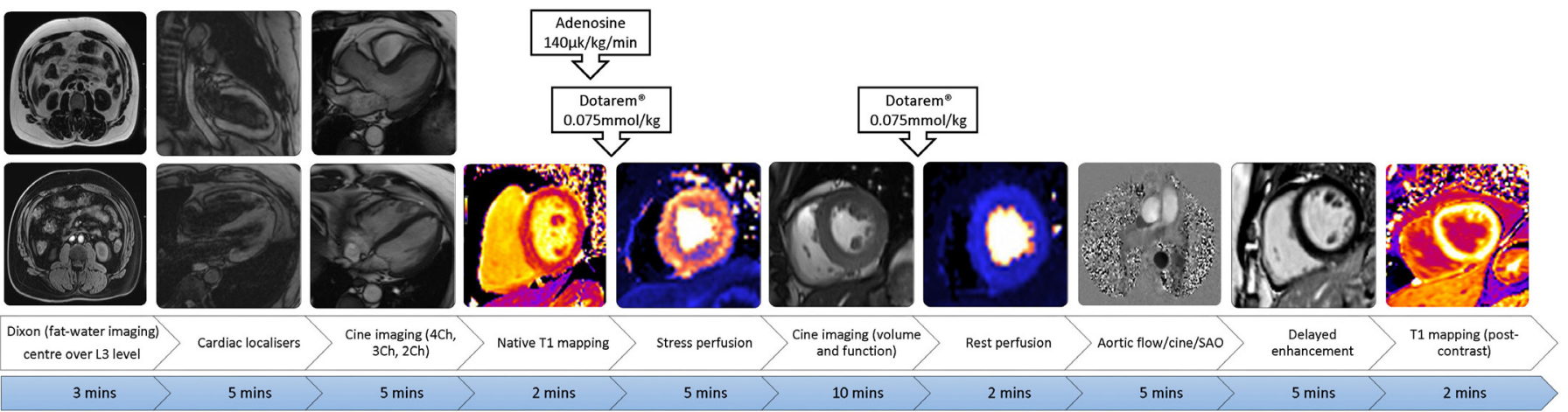

Patients will be fully counselled about the procedure (which may cause slight discomfort and claustrophobia) and written consent gained prior to the MRI scan. A standard MRI safety questionnaire will be completed prior to the scan to ensure the safety requirements are satisfied. A 12-lead ECG will be performed within 72 hours prior to the cardiac MRI scan.

Two intravenous cannulae will be inserted for administration of adenosine or dobutamine and gadolinium contrast agent. Patients may receive intravenous sedation if they are anxious or claustrophobic. Adenosine will be administered at $140 \mu \mathrm{g} / \mathrm{kg} / \mathrm{min}$, limited to $3-4 \mathrm{~min}$ to minimise patient discomfort. If there is no haemodynamic response and/or the patient does not experience any effects, the infusion may be prolonged or the dose may be increased incrementally to $210 \mu \mathrm{g} / \mathrm{kg} / \mathrm{min}$ (at the discretion of the supervising physician). In patients with an absolute contraindication to adenosine (severe asth$\mathrm{ma} /$ chronic obstructive pulmonary disease, high degree of atrioventricular block, theophylline usage), dobutamine/atropine will be administered as the stressor agent. The dose of dobutamine will commence at $20 \mu \mathrm{g} / \mathrm{kg} /$ min for those with preserved ejection $(10 \mu \mathrm{g} / \mathrm{kg} / \mathrm{min}$ for patients with significant LV dysfunction) and increased incrementally until age-predicted target heart rate is reached ( $\geq 85 \%$ of 220 -age beats per minute). It is anticipated the maximum dose administered will be $30 \mu \mathrm{g} /$ $\mathrm{kg} / \mathrm{min} .{ }^{57}$ Atropine, $0.6-1.8 \mathrm{mg}$, may be used to achieve predicted maximum heart rate. Chest pain, arrhythmias and induction of wall motion abnormality are indications to stop the infusion, and a patient's heart rate and blood pressure will be monitored closely. Patients will be reassured throughout and it will be made clear they can stop the MRI scan at any time.

Cardiac MR analysis will be both qualitative and quantitative. Local on-site reporting will include any regional motion abnormality by visual analysis using the 17 -segment American Heart Association model ${ }^{58}$ and each segment will be scored as 0 (normal), 1 (mild hypokinesia), 2 (severe hypokinesia), 3 (akinesia) or 4 (dyskinesia). Hypoperfusion (ischaemia) will be assessed by a visual comparison of stress and rest cardiac MR perfusion scan in 16 of the 17 AHA model segments (excluding the apical cap segment) with scores of 0 (normal), 1 (equivocal), 2 (subendocardial ischaemia) or 3 (transmural ischaemia). Any infarct (scar) will be reported based on the late gadolinium-enhancement images (17 segment AHA model) with scores of 0 (none), 1 (1\%-25\%), 2 (26\%-50\%), 3 $(51 \%-75 \%)$ or $4(>75 \%)$ for each segment. Patients who undergo open surgical revascularisation or major lower limb amputation (major surgery) will undergo an additional follow-up scan at 2-4 months postoperatively to investigate for incidence of perioperative MI. ${ }^{59}$

All patients undergoing cardiac MRI will be consented to have an additional axial image at the level of the third lumbar vertebrae during the initial scan only, which will be performed before the cardiac sequences. This will image psoas, paraspinal and abdominal wall muscles allowing the total skeletal muscle area to be measured as an assessment of sarcopenia. ${ }^{60}$

\section{Biomarkers additional assessments}

Venepuncture will be performed by a qualified member of the research team and blood collected using an S-Monovette $9 \mathrm{~mL}$ K2 EDTA blood tube and an S-Monovette $7.5 \mathrm{~mL}$ Serum Gel blood tube with the aim of collecting $6 \times 500 \mu \mathrm{L}$ aliquots of both serum and plasma. All samples will be stored at the NIHR Leicester Biomedical Research Centre located at Glenfield Hospital for subsequent analysis. Consent will be gained to use these samples in future biomarker analyses. All samples will be anonymously datalinked with clinical baseline and outcome data for patients recruited to the biomarkers additional assessments.

\section{Study assessment schedule}

An overview of study procedures and assessment schedule is detailed in table 2 .

\section{Definition of end of study}

The end of study date is defined by the date the long-term outcomes are collected from NHS Digital, which will be ongoing for 10 years following the date the final patient is recruited to the primary cohort.

\section{Discontinuation/withdrawal from the study}

A patient, or personal consultee, may withdraw from the study at any point without giving any reason with no 
Table 2 Study procedures and patient assessment schedule

\begin{tabular}{|c|c|c|c|c|c|c|c|}
\hline Procedure/assessment & Baseline & $\begin{array}{l}24 \text { hours } \\
\text { postintervention }\end{array}$ & $\begin{array}{l}72 \text { hours } \\
\text { postintervention }\end{array}$ & $\begin{array}{l}\text { 3-month } \\
\text { F/U visit }\end{array}$ & $\begin{array}{l}\text { 12-month } \\
\text { F/U visit }\end{array}$ & $\begin{array}{l}\text { 12-month } \\
\text { telephone } F / U\end{array}$ & $\begin{array}{l}24 \text { month } \\
\text { telephone } F / U\end{array}$ \\
\hline $\begin{array}{l}\text { Patient consent/ consultee } \\
\text { declaration }\end{array}$ & $\bullet$ & & & & & & \\
\hline Clinical frailty scale & $\bullet$ & & & $\bullet$ & $\bullet$ & $\bullet$ & $\bullet$ \\
\hline Barthel Index & $\bullet$ & & & $\bullet$ & $\bullet$ & • & $\bullet$ \\
\hline VascuQoL & • & & & • & $\bullet$ & • & • \\
\hline Edmonton frail scale & ○ & & & $\bullet$ & $\bullet$ & & \\
\hline MoCA & $\bullet$ & & & $\bullet$ & $\bullet$ & & \\
\hline SPPB & $\bullet$ & & & $\bullet$ & $\bullet$ & & \\
\hline Grip strength & $\bullet$ & & & $\bullet$ & $\bullet$ & & \\
\hline Blood sample collection & 0 & & & & & & \\
\hline
\end{tabular}

Additional assessments: Green: frailty and cognitive; purple: cardiac MRI; red: biomarkers.

4AT, 4 'A's Test (4AT) for rapid delirium screening; HADS, Hospital Anxiety and Depression Scale; MoCA, Montreal Cognitive Assessment; MRI, Magnetic Resonance Imaging; SPPB, Short Physical Performance Battery; SQiD, Single Question in Delirium; VascuQoL, Vascular Quality of Life questionnaire.

effect to their medical care or legal rights. This will be explained at recruitment and each follow-up contact. If the patient (or personal consultee) withdrawing from the study voluntarily offers a reason for their withdrawal, this will be documented in the medical notes and collected as part of the research data. Patient data collected up to the point of withdrawal will be used in the analysis unless the patient (or personal consultee) has withdrawn consent for all data to be used. Similarly, if they withdraw consent for blood samples to be used prior to analysis, these will be destroyed. Long-term data will be collected via datalinkage unless consent is withdrawn.

\section{Study patient and public involvement (PPI) group}

A PPI group involving patients, and their carers, with experience of being treated for SLI at the Leicester Vascular Institute has been established for this study. Members of the PPI group have been directly involved in the development of the study, the information material and provided insight on the burden of the study from a patient's perspective. The group will continue to meet throughout the duration of the study to be informed of the progress and have the opportunity to advise regarding the ongoing delivery of the study.

\section{Planned study dates}

The study began on 10 May 2019 and recruitment will continue until 9 May 2021. The study will end on 9 May 2031 after completion of 10-year follow-up via NHS Digital.

\section{DATA AND ANALYSIS}

Study data will be collected and managed using REDCap (Research Electronic Data Capture) hosted at the
University of Leicester. ${ }^{61}$ REDCap is a secure, web-based application designed to support data capture for research studies.

A full statistical analysis plan will be written and agreed by all investigators before database lock. A range of continuous and categorical data will be collected at baseline and at various time points on the patients recruited into the primary cohort and the additional assessments. Descriptive statistics of the baseline data will be presented using appropriate tabular and graphical methods both for the prospective cohort and the historical controls. Comparability of the cohorts in terms of baseline characteristics will be assessed using appropriate statistical tests, for example, t-tests for continuous variables and $\chi^{2}$ tests for categorical variables.

The proportion of patients undergoing a major amputation within the first 12 months will be calculated and presented with $95 \%$ CI. The prospective cohort will be compared with the historical controls using logistic regression with major amputation within 12 months as the dependent variable. Given there maybe systematic differences between the prospective and historical cohorts, adjustment for propensity score will be used. The propensity score model will include all baseline characteristics available in both cohorts as independent variables with cohort (prospective/historical) as the dependent variable. The final form of the propensity score model will be specified in the statistical analysis plan prior to analysis commencing. The model will be run both unadjusted and adjusted for the propensity score. An adjustment for propensity score, rather than propensity score matching, will be used to ensure that all participants are included in the analysis. ${ }^{62} 63$ 
A similar modelling strategy will be employed to assess the association between interventions (amputation vs no amputation) and short-term, medium-term and longterm outcomes. Cox proportional hazards modelling will be used to assess association with both mortality and amputation-free survival. The prevalence $(95 \% \mathrm{CI})$ will also be calculated for frailty, cognitive impairment and cardiac disease (detected by stress MRI).

The assumptions of all models fitted will be assessed. The levels and reasons for missing data for all key outcomes and confounders will be assessed. Where more than $5 \%$ of data are missing, multiple imputation will be used. Where less than 5\% are missing, a complete case analysis will be performed.

Statistical analyses will be two-sided with a $5 \%$ significance level.

\section{Sample size calculation}

The historical amputation rate among patients with SLI is around 25\%. ${ }^{4}$ We anticipate a reduction in amputation rate among this contemporary cohort in the region of $10 \%$. A sample size of 335 patients will be required to detect a $10 \%$ reduction in amputation rate in comparison to a similar sized historical cohort, with $90 \%$ power and type I error rate of $5 \%$. The target sample size will be 420 patients to account for a potential loss to follow-up of $20 \%$. A minimum of 335 patients in the contemporary cohort will enable an estimate of amputation rate of $15 \%$ with a $95 \%$ CI of $11.3 \%$ to $19.2 \%$, which is clinically acceptable.

\section{ETHICS AND DISSEMINATION}

This study has received ethical approval from the UK National Research Ethics Service (19/LO/0132). The study sponsor is the University of Leicester and all their standard operating procedures will be followed ensuring this study complies with all relevant legislation and guidelines. Direct access will be granted to authorised representatives from the sponsor, host institution and regulatory authorities to permit study-related monitoring, audit and inspection. The chief investigator (RDS) will ensure that this study is conducted in full conformity with the Declaration of Helsinki (last amended October 2013).

As this is not considered a high-risk study, adverse events or adverse reactions will not be reported. There are many serious adverse events (SAEs) that are reasonably expected in this group of patients. Expected SAEs (online supplementary file 1) that are unrelated to the study will not be reported. All unexpected SAEs and serious adverse reactions related to the study will be documented on the SAE form and reported to the sponsor within 24 hours of the investigator becoming aware of the event.

A summary of findings will be available to patients on request. It is the intention to disseminate any findings from the study at relevant national and international meetings and in peer-reviewed medical journals. Results will also be distributed through social media and in plain language using our University accounts to increase access to the general public. All clinicians and researchers involved will be acknowledged in written papers. For each output, a writing team will be convened from the study group and external collaborators (where necessary). Authorship will be decided on a paper-by-paper basis. Anonymised study data will be available on request to the chief investigator (RDS).

\section{DISCUSSION}

The results of this study should provide a valuable update to current observational evidence regarding contemporary outcomes of patients with SLI. The additional assessments will also provide useful observational evidence, which may help risk-stratify patients both in terms of risk of amputation and mortality based on frailty, cognitive impairment and cardiac MRI findings. Future biomarker analyses of samples collected during the study may also provide evidence for established and novel biomarkers in the risk stratification of patients with SLI. The facility to include patients without capacity to consent will reduce selection bias and should provide far more representative data particularly regarding older patients with SLI, and the prevalence of frailty and cognitive impairment in this cohort.

\section{Limitations}

There are a number of limitations to this study. As this is a single-centre study, the generalisability of the results will be limited, particularly as amputation rate will be impacted on by local management strategies and patient demographics. Additionally, there is not the resource to provide for recruitment of non-English-speaking patients. While we do not anticipate significant numbers of patients will be excluded for this reason, as Leicester has an ethnically diverse population, results from this study may not be reflective of the whole population.

Prospective data have not been collected for patients with SLI presenting prior to the establishment of the rapid-access limb salvage clinic and improved SLI and DFD management pathways: subsequently, data will be retrospectively collected for a historical cohort to compare amputation rates. Any observed differences in amputation rates may therefore be due to factors other than modern revascularisation techniques and improved care pathways. Statistical analyses with adjustment for propensity score may mitigate this to a degree; however, any observed effect may still be liable to unidentified confounding. Furthermore, retrospective data collected will not be as reliable as the data from the prospective cohort, limiting the comparison between the two cohorts; however, it is difficult to determine whether this may underestimate or overestimate the amputation rate in the historical cohort prior to completing historical data collection. Frailty, cognitive function, cardiac MRI and biomarker analysis will not be available for patients in the historical cohort so no comparisons will be possible. 


\section{CONCLUSION}

The LIMb (leg ischaemia management collaboration) study will report the current amputation rate in patients presenting with severe limb ischaemia. It aims to quantify the impact of a rapid-access vascular limb salvage clinic and improved management pathways on amputation rate, and the potential role for cardiac MR, frailty and cognitive assessments, and biomarker analysis in risk-stratifying patients undergoing intervention for SLI.

\section{Author affiliations}

${ }^{1}$ Department of Cardiovascular Sciences, University of Leicester, Leicester, UK ${ }^{2}$ Leicester Vascular Institute, University Hospitals of Leicester NHS Trust, Leicester, UK

${ }^{3}$ National Institute for Health Research Leicester Biomedical Research Centre - The Glenfield Hospital, Leicester, UK

${ }^{4}$ Department of Health Sciences, University of Leicester, Leicester, UK

${ }^{5}$ Cardiac/Pulmonary Rehabilitation, University Hospitals of Leicester NHS Trust, Leicester, UK

Acknowledgements The authors would like to thank George Davies and the George Davies Charitable Trust for the generous charitable donation that funded this study, Natasha Bryant for the development of the data capture and management procedures, and Gaurav Gulsin for providing the research images of the cardiac MR sequence.

Contributors All authors contributed to the study design. JSMH contributed to writing the study protocol and drafted and revised the paper. SN, AN, SS, SC, VH and GPM contributed to writing the study protocol and revised the draft paper. TJP is the study manager, edited the study protocol and revised the draft paper. SS wrote the data analysis plan and study database, and revised the draft paper. MN and LG wrote the statistical analysis plan and revised the draft paper. GPM, HR, TR and $M B$ revised the draft paper. RSMD is the principal investigator and revised the draft paper. RDS is the chief investigator, wrote the study protocol and revised the draft paper.

Funding The LIMb study and LIMb investigators JSMH, AN, SN and TJP are funded, and RDS part-funded, by a charitable donation from the George Davies Charitable Trust (Registered Charity Number: 1024818) and supported by the National Institute for Health Research (NIHR) Leicester Biomedical Research Centre. GPM is funded by an NIHR Research Professorship (2017-08-ST2-007). TR is an NIHR senior investigator.

Competing interests RSMD has acted as a speaker for Gore Medical and has received educational grants from Terumo/Aortic outside the submitted work.

Patient consent for publication Not required.

Ethics approval Ethical approval for this study has been granted by the UK National Research Ethics Service (19/L0/0132).

Provenance and peer review Not commissioned; externally peer reviewed.

Open access This is an open access article distributed in accordance with the Creative Commons Attribution Non Commercial (CC BY-NC 4.0) license, which permits others to distribute, remix, adapt, build upon this work non-commercially, and license their derivative works on different terms, provided the original work is properly cited, appropriate credit is given, any changes made indicated, and the use is non-commercial. See: http://creativecommons.org/licenses/by-nc/4.0/.

\section{REFERENCES}

1. Fowkes FGR, Rudan D, Rudan I, et al. Comparison of global estimates of prevalence and risk factors for peripheral artery disease in 2000 and 2010: a systematic review and analysis. The Lancet 2013;382:1329-40.

2. Criqui $\mathrm{MH}$, Aboyans $\mathrm{V}$. Epidemiology of peripheral artery disease. Circ Res 2015;116:1509-26.

3. Becker F, Robert-Ebadi H, Ricco J-B, et al. Chapter I: definitions, epidemiology, clinical presentation and prognosis. European Journal of Vascular and Endovascular Surgery 2011;42(Suppl 2):S4-S12.
4. Norgren L, Hiatt WR, Dormandy JA, et al. Inter-Society consensus for the management of peripheral arterial disease (TASC II). J Vasc Surg 2007;45 Suppl S:S5-67.

5. Ahmad N, Thomas GN, Gill P, et al. The prevalence of major lower limb amputation in the diabetic and non-diabetic population of England 2003-2013. Diabetes and Vascular Disease Research 2016;13:348-53.

6. Kerr M. Diabetic foot care in England: an economic study: diabetes UK, 2017. Available: https://diabetes-resources-production. s3-eu-west-1.amazonaws.com/diabetes-storage/migration/pdf/ Diabetic\%2520footcare\%2520in\%2520England\%2C\%2520An\% 2520 economic\%2520case\%2520study\%2520\%28January\% 25202017\%29.pdf

7. Jeffcoate W, Bakker K. World diabetes day: footing the bill. The Lancet 2005;365.

8. Davie-Smith F, Coulter E, Kennon B, et al. Factors influencing quality of life following lower limb amputation for peripheral arterial occlusive disease: a systematic review of the literature. Prosthet Orthot Int 2017;41:537-47.

9. Suckow BD, Goodney PP, Cambria RA, et al. Predicting functional status following amputation after lower extremity bypass. Ann Vasc Surg 2012;26:67-78.

10. Naylor H, Russell P, Thompson J. Development of a scoring tool (BLARt score) to predict functional outcome in lower limb amputees AU - Bowrey, Sarah. Disability and Rehabilitation 2018:1-9.

11. Takeji Y, Yamaji K, Tomoi Y, et al. Impact of frailty on clinical outcomes in patients with critical limb ischemia. Circulation 2018;11:e006778.

12. Hubbard RE, Story DA. Patient frailty: the elephant in the operating room. Anaesthesia 2014;69(Suppl 1):26-34.

13. Morley JE, Vellas B, Abellan van Kan G, et al. Frailty consensus: a call to action. J Am Med Dir Assoc 2013;14:392-7.

14. Gale CR, Cooper C, Aihie Sayer A. Prevalence of frailty and disability: findings from the English longitudinal study of ageing. Age Ageing 2015;44:162-5.

15. Partridge JSL, Fuller M, Harari D, et al. Frailty and poor functional status are common in arterial vascular surgical patients and affect postoperative outcomes. Int J Surg 2015;18:57-63.

16. Xue Q-L. The frailty syndrome: definition and natural history. Clin Geriatr Med 2011;27:1-15.

17. Wang J, Zou Y, Zhao J, et al. The impact of frailty on outcomes of elderly patients after major vascular surgery: a systematic review and meta-analysis. Eur J Vasc Endovasc Surg 2018;56:591-602.

18. de Vries NM, Staal JB, van Ravensberg CD, et al. Outcome instruments to measure frailty: a systematic review. Ageing Res Rev 2011;10:104-14.

19. Robertson DA, Savva GM, Kenny RA. Frailty and cognitive impairment-A review of the evidence and causal mechanisms. Ageing Res Rev 2013;12:840-51.

20. Partridge JSL, Dhesi JK, Cross JD, et al. The prevalence and impact of undiagnosed cognitive impairment in older vascular surgical patients. J Vasc Surg 2014;60:1002-11.

21. Fried LP, Tangen CM, Walston J, et al. Frailty in older adults: evidence for a phenotype. The Journals of Gerontology Series A Biological Sciences and Medical Sciences 2001;56:M146-M157.

22. Cruz-Jentoft AJ, Bahat G, Bauer J, et al. Sarcopenia: revised European consensus on definition and diagnosis. Age Ageing 2019;48:16-31

23. Reeve TE, Ur R, Craven TE, et al. Grip strength measurement for frailty assessment in patients with vascular disease and associations with comorbidity, cardiac risk, and sarcopenia. $J$ Vasc Surg 2018:67:1512-20.

24. Matsubara $\mathrm{Y}$, Matsumoto $\mathrm{T}$, Inoue $\mathrm{K}$, et al. Sarcopenia is a risk factor for cardiovascular events experienced by patients with critical limb ischemia. J Vasc Surg 2017;65:1390-7.

25. Pol RA, van Leeuwen BL, Visser L, et al. Standardised frailty indicator as predictor for postoperative delirium after vascular surgery: a prospective cohort study. European Journal of Vascular and Endovascular Surgery 2011;42:824-30.

26. Visser L, Prent A, van der Laan MJ, et al. Predicting postoperative delirium after vascular surgical procedures. J Vasc Surg 2015;62:183-9 https://doi.org/

27. Hertzer NR. Clinical experience with preoperative coronary angiography. J Vasc Surg 1985;2:510-2.

28. Her K, Choi C, Park Y, et al. Concomitant peripheral artery disease and asymptomatic coronary artery disease: a management strategy. Ann Vasc Surg 2008;22:649-56.

29. Pande RL, Perlstein TS, Beckman JA, et al. Secondary prevention and mortality in peripheral artery disease: National health and nutrition examination study, 1999 to 2004. Circulation 2011;124:17-23. 
30. Landesberg G, Mosseri M, Wolf YG, et al. Preoperative thallium scanning, selective coronary revascularization, and long-term survival after major vascular surgery. Circulation 2003;108:177-83.

31. Wagner A, Mahrholdt H, Holly TA, et al. Contrast-Enhanced MRI and routine single photon emission computed tomography (SPECT) perfusion imaging for detection of subendocardial myocardial infarcts: an imaging study. The Lancet 2003;361:374-9.

32. Khoo JP, Grundy BJ, Steadman CD, et al. Stress cardiovascular Mr in routine clinical practice: referral patterns, accuracy, tolerance, safety and incidental findings. Br J Radiol 2012;85:e851-7.

33. Greenwood JP, Ripley DP, Berry C, et al. Effect of care guided by cardiovascular magnetic resonance, myocardial perfusion scintigraphy, or NICE guidelines on subsequent unnecessary angiography rates. JAMA 2016;316:1051-60.

34. Greenwood JP, Maredia N, Younger JF, et al. Cardiovascular magnetic resonance and single-photon emission computed tomography for diagnosis of coronary heart disease (CE-MARC): a prospective trial. The Lancet 2012;379:453-60.

35. Xue H, Inati S, Sørensen TS, et al. Distributed MRI reconstruction using Gadgetron-based cloud computing. Magn Reson Med 2015;73:1015-25.

36. Kellman P, Hansen MS, Nielles-Vallespin S, et al. Myocardial perfusion cardiovascular magnetic resonance: optimized dua sequence and reconstruction for quantification. Journal of Cardiovascular Magnetic Resonance 2017;19.

37. Engblom H, Xue H, Akil S, et al. Fully quantitative cardiovascular magnetic resonance myocardial perfusion ready for clinical use: a comparison between cardiovascular magnetic resonance imaging and positron emission tomography. Journal of Cardiovascular Magnetic Resonance 2017;19.

38. Mills JL, Conte MS, Armstrong DG, et al. The Society for vascular surgery lower extremity threatened limb classification system: risk stratification based on wound, ischemia, and foot infection (WIfI). $J$ Vasc Surg 2014;59:220-34.

39. Aboyans V, Ricco J-B, Bartelink M-LEL, et al. 2017 ESC Guidelines on the Diagnosis and Treatment of Peripheral Arterial Diseases, in collaboration with the European Society for Vascular Surgery (ESVS): Document covering atherosclerotic disease of extracranial carotid and vertebral, mesenteric, renal, upper and lower extremity arteriesEndorsed by: the European Stroke Organization (ESO) The Task Force for the Diagnosis and Treatment of Peripheral Arterial Diseases of the European Society of Cardiology (ESC) and of the European Society for Vascular Surgery (ESVS). Eur Heart $J$ 2018;39:763-816.

40. Breen P, Simpson S, Packer C. New and emerging angioplasty technologies in development for severe lower limb ischaemia. Birmingham, UK: The NIHR Horizon Scanning Research \& Intelligence Centre University of Birmingham, 2016.

41. Slim H, Tiwari A, Ahmed A, et al. Distal versus ultradistal bypass grafts: amputation-free survival and patency rates in patients with critical leg ischaemia. Eur J Vasc Endovasc Surg 2011;42:83-8.

42. Taeymans K, Groot Jebbink E, Holewijn S, et al. Three-Year outcome of the covered endovascular reconstruction of the aortic bifurcation technique for aortoiliac occlusive disease. J Vasc Surg 2018;67:1438-47 https://doi.org/

43. Boyle J, Brooks M, Biram R, et al. A Best Practic Clinical Care Pathway for Peripheral Arterial Disease London, UK: The Vascular Society of Great Britain \& Ireland, 2019. Available: https://www. vascularsociety.org.uk/_userfiles/pages/files/Newsletters/PAD\% 20QIF\%20April\%202019(1).pdf [Accessed 12 Ap 2019].

44. Edmans J, Bradshaw L, Gladman JRF, et al. The identification of seniors at risk (ISAR) score to predict clinical outcomes and health service costs in older people discharged from UK acute medical units. Age Ageing 2013;42:747-53.

45. Adam DJ, Beard JD, Cleveland T, et al. Bypass versus angioplasty in severe ischaemia of the leg (basil): multicentre, randomised controlled trial. Lancet 2005;366:1925-34.

46. Ince P, Abbas ZG, Lutale JK, et al. Use of the Sinbad classification system and score in comparing outcome of foot ulcer management on three continents. Diabetes Care 2008;31:964-7.

47. Rockwood Ket al. A global clinical measure of fitness and frailty in elderly people. Can Med Assoc J 2005;173:489-95.

48. Morgan MBF, Crayford T, Murrin B, et al. Developing the vascular quality of life questionnaire: a new disease-specific quality of life measure for use in lower limb ischemia. J Vasc Surg 2001;33:679-87.

49. Collin C, Wade DT, Davies S, et al. The Barthel ADL index: a reliability study. Int Disabil Stud 1988;10:61-3.

50. Zigmond AS, Snaith RP. The hospital anxiety and depression scale. Acta Psychiatr Scand 1983;67:361-70.

51. Rolfson DB, Majumdar SR, Tsuyuki RT, et al. Validity and reliability of the Edmonton frail scale. Age Ageing 2006;35:526-9.

52. Nasreddine ZS, Phillips NA, Bédirian V, et al. The Montreal cognitive assessment, MoCA: a brief screening tool for mild cognitive impairment. J Am Geriatr Soc 2005;53:695-9.

53. Guralnik JM, Simonsick EM, Ferrucci L, et al. A short physical performance battery assessing lower extremity function: association with self-reported disability and prediction of mortality and nursing home admission. J Gerontol 1994;49:M85-M94.

54. Sands MB, Dantoc BP, Hartshorn A, et al. Single question in delirium (SQiD): testing its efficacy against psychiatrist interview, the confusion assessment method and the Memorial delirium assessment scale. Palliat Med 2010;24:561-5.

55. Bellelli G, Morandi A, Davis DHJ, et al. Validation of the 4AT, a new instrument for rapid delirium screening: a study in 234 hospitalised older people. Age Ageing 2014;43:496-502.

56. Singh A, Greenwood JP, Berry C, et al. Comparison of exercise testing and CMR measured myocardial perfusion reserve for predicting outcome in asymptomatic aortic stenosis: the prognostic importance of microvascular dysfunction in aortic stenosis (PRIMID as) study. Eur Heart J 2017;38:1222-9.

57. Mordi I, Stanton T, Carrick D, et al. Comprehensive dobutamine stress CMR versus echocardiography in LBBB and suspected coronary artery disease. JACC Cardiovasc Imaging 2014;7:490-8.

58. Cerqueira MD, Weissman NJ, Dilsizian V, et al. Standardized myocardial segmentation and nomenclature for tomographic imaging of the heart. A statement for healthcare professionals from the cardiac imaging Committee of the Council on clinical cardiology of the American heart association. Circulation 2002;105:539-42.

59. Rahimi K, Banning AP, Cheng ASH, et al. Prognostic value of coronary revascularisation-related myocardial injury: a cardiac magnetic resonance imaging study. Heart 2009;95:1937-43.

60. Kays JK, Liang TW, Zimmers TA, et al. Sarcopenia is a significant predictor of mortality after abdominal aortic aneurysm repair. JCSM Clinical Reports 2018;3.

61. Harris PA, Taylor R, Thielke R, et al. Research electronic data capture (REDCap)-A metadata-driven methodology and workflow process for providing translational research informatics support. J Biomed Inform 2009:42:377-81.

62. Austin PC. An introduction to propensity score methods for reducing the effects of confounding in observational studies. Multivariate Behav Res 2011;46:399-424.

63. Lim J, Walley R, Yuan J, et al. Minimizing patient burden through the use of historical Subject-Level data in innovative confirmatory clinical trials. Drug Inf J 2018;52:546-59. 